\title{
Operational Regimes in Picosecond and Femtosecond Pulse-Excited Ultrahigh Vacuum SERS
}

Eric A. Pozzi,,$^{\dagger}$ Natalie L. Gruenke, ${ }^{\dagger}$ Naihao Chiang, ${ }^{\S}$ Dmitry V. Zhdanov, ${ }^{\dagger}$ Nan Jiang, ${ }^{\dagger}, "$ Tamar Seideman, ${ }^{\dagger,}$ George C. Schatz, ${ }^{\dagger}$ Mark C. Hersam, ${ }^{\dagger, t,}$ and Richard P. Van Duyne ${ }^{\dagger, \xi, *}$

${ }^{\dagger}$ Department of Chemistry, ${ }^{\dagger}$ Department of Materials Science and Engineering, and ${ }^{\S}$ Applied Physics Program, Northwestern University, Evanston, IL 60208, United States

Supporting Information

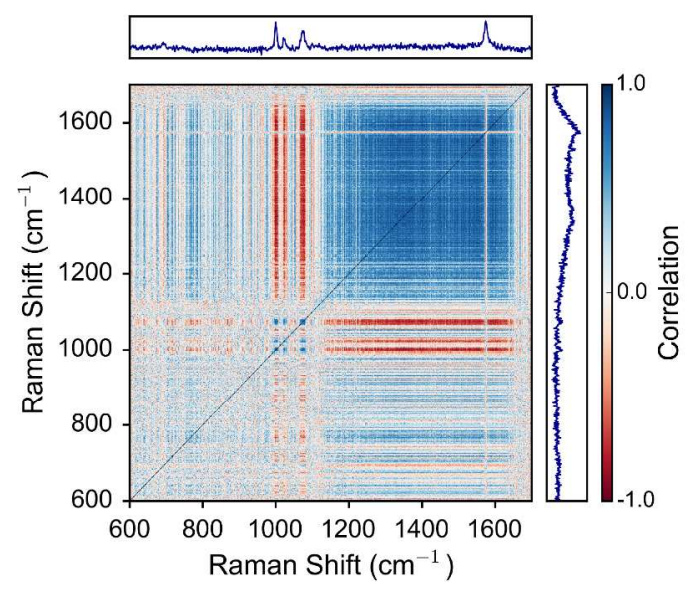

Figure S1: 2D plot of the statistical correlation between BT SERS intensity at each pixel among spectra before and after one $10 \mathrm{~s}$ irradiation of the fs 485 beam with pulse energy density $9.9 \times 10^{-}$ ${ }^{4} \mathrm{~J} \mathrm{~cm}^{-2}$ pulse $^{-1}$. BT SERS peaks and carbonaceous background signals are strongly anticorrelated with one another, suggestive of a molecular decay mechanism. BT and amorphous carbon background spectra are displayed above and to the right of the correlation plot, respectively, for reference. 


\section{Estimation of the heating effect}

In this section we will calculate the upper bound for temperature increase caused by laser exposure.

\section{Instantaneous heating by a single pulse.}

We will consider a rough but secure upper bound for the heating effect, assuming that all the energy of the femtosecond pulses is converted into heat in the silver film. In other words, we will completely neglect reflection and scattering. We will also use a step-like spatial heating profile assuming that the incoming laser energy flux is uniformly distributed inside a spot of characteristic radius $R \sim 25 \mu \mathrm{m}$ and is zero outside (this assumption will enable us to obtain analytical solutions of the heat equation). Under these assumptions, the temperature change $\delta T_{\text {pulse }}$ in the exposed area immediately after a femtosecond pulse can be estimated as

$$
\delta \mathrm{T}_{\text {pulse }}=\frac{E_{e}}{c_{s} \rho_{s} l}
$$

where $\rho_{\mathrm{s}}$ and $c_{\mathrm{s}}$ are the silver density and heat capacity, $l \sim 250 \mathrm{~nm}$ is the film thickness and $E_{\mathrm{e}}$ is the energy density per pulse. The resulting magnitudes of the instantaneous heating effect for various pulse energies are shown in Fig. S2. For maximal experimental energy density $E_{\mathrm{e}} \sim 10^{-3}$ $\mathrm{J} / \mathrm{cm}^{2}$ the estimated temperature change is $\delta T_{\text {pulse }} \sim 15 \mathrm{~K}$.

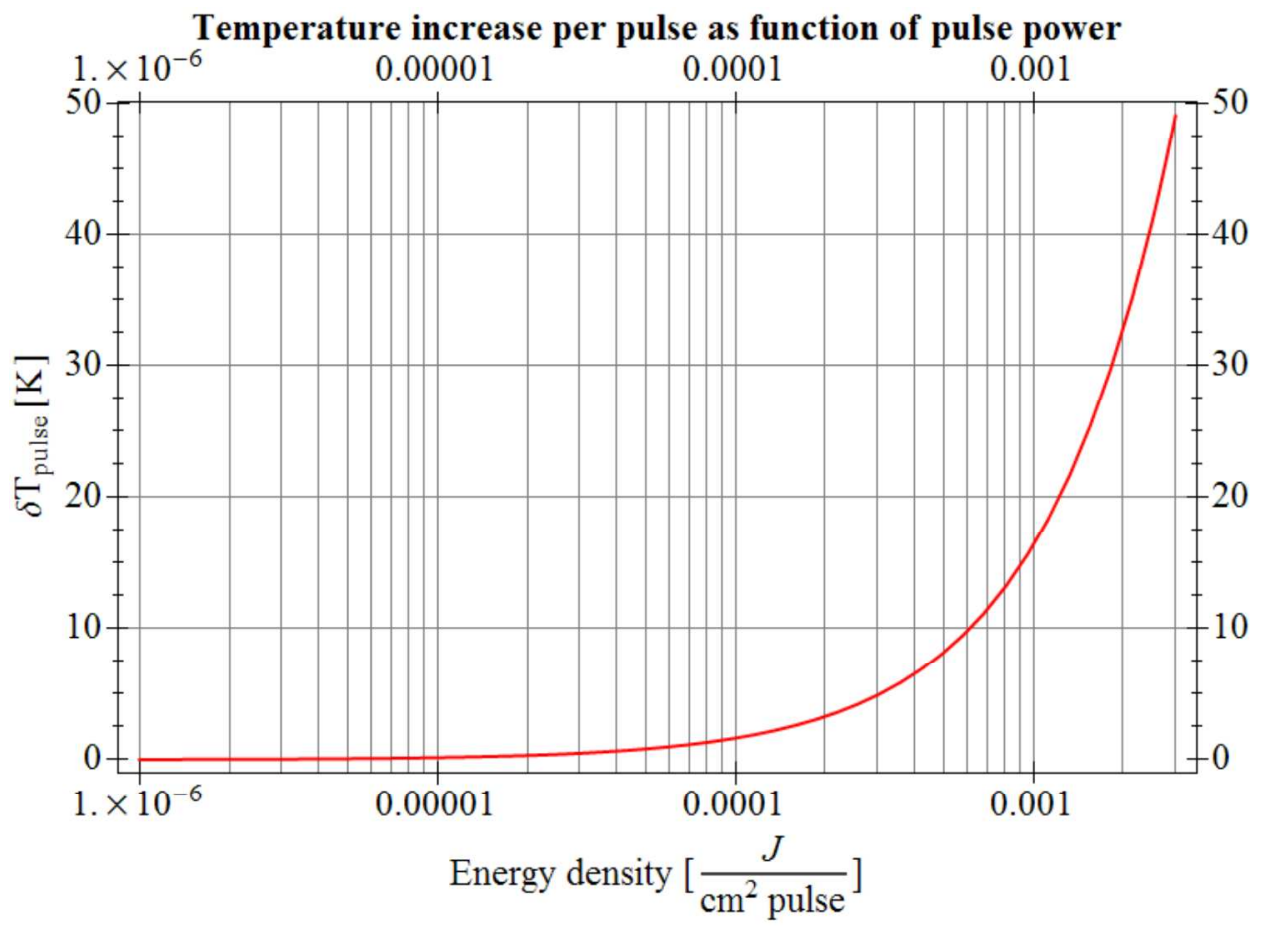

Fig. S2. Local heating induced by a single pulse as a function of pulse energy density. 
2. Heating effect at experimental conditions.

The actual laser-induced temperature change $\Delta \mathrm{T}$ results from the accumulated contributions of a large number of pulses and the competing effect of heat dissipation. It is convenient to express $\Delta \mathrm{T}$ as

$$
\Delta \mathrm{T}=\xi \delta T_{\text {pulse }} .
$$

In what follows we will call $\xi$ the amplification coefficient.

There are two major dissipation channels in our experiment:

I) Dissipation along the silver film;

II) Dissipation into the silica substrate.

The upper bound for the amplification coefficient can be estimated as $\xi<\min \left(\xi_{\mathrm{b}} \xi_{\mathrm{II}}\right)$, where $\xi_{k}$ is the amplification coefficient calculated with the assumption that only the $k$-th dissipation channel is present.

In order to estimate $\xi_{\mathrm{I}}$ we will neglect the heat dissipation into the substrate and approximate the silver layer as an infinite flat film. The effect of film roughness will be included phenomenologically by introducing a reduced effective heat conductance coefficient: $\beta_{\mathrm{eff}} \sim \beta_{\mathrm{s}} / 2$, where $\beta_{\mathrm{s}}$ is the heat conductance of silver. This model leads to the heat equation, which has cylindrical symmetry:

$$
\alpha \frac{\partial}{\partial t} \delta \mathrm{T}-\beta \frac{1}{r} \frac{\partial}{\partial r}\left(r \frac{\partial}{\partial r} \delta \mathrm{T}\right)=0,
$$

where $r$ is the distance from the center of the laser spot and $\alpha=c_{\mathrm{s}} \rho_{\mathrm{s}}$.

Consider first the time-dependent temperature changes $\delta T_{\text {pulse }}(r, t)$ caused by a single femtosecond laser pulse applied at $t=0$. Under all assumptions made, eq. (S1) has the following closed-form solution for the temperature at the hottest point $(r=0)$ :

$$
\delta \mathrm{T}_{\text {pulse }}(0, t)=\delta \mathrm{T}_{\text {pulse }}(0,0)\left(1-e^{-\frac{\alpha R^{2}}{4 \beta_{\text {eff }} t}}\right),
$$

Eq. (S2) allows us to estimate the amplification coefficient $\xi_{\text {I }}$ corresponding to the heating effect of the femtosecond pulse combined with repetition rate $1 / \delta t$ :

$$
\xi=\frac{1}{\delta T_{\text {pulse }}(0,0)} \sum_{n=0}^{\infty} \delta \mathrm{T}\left(0, \delta \mathrm{t}_{\text {pulse }} n\right)
$$

Substituting the experimental value $\delta t=10^{-5} \mathrm{~s}$ into (S3) one obtains the estimate $\xi_{\mathrm{r}} \sim 3.6$.

Let us now turn to the calculation of $\xi_{\mathrm{II}}$. In order to obtain an analytical solution for this case we will use the simplified model shown in Fig. S3. 


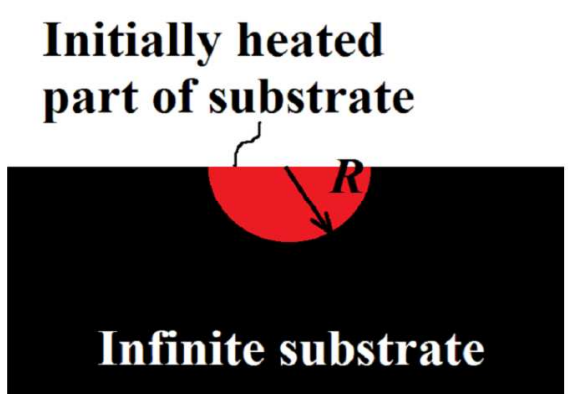

Fig. S3. Simplified model for estimating $\xi_{\mathrm{II}}$.

Specifically, we replace the thin laser-heated silver layer with the hemisphere of substrate heated to the same temperature. Note that this replacement will give us overestimated temperature both due to larger heated volume, larger heat capacitance and lower heat conductance of silica compared to silver. To roughly account for these factors we should use $R_{\mathrm{eff}}=R / 2 \ldots R / 4$.

This model allows us to cast the heat equation into an effective spherically symmetric form:

$$
\alpha_{\mathrm{sc}} \frac{\partial}{\partial t} \delta \mathrm{T}-\beta_{\mathrm{sc}} \frac{1}{r^{2}} \frac{\partial}{\partial r}\left(r^{2} \frac{\partial}{\partial r} \delta \mathrm{T}\right)=0
$$

where $\alpha_{\mathrm{sc}}=c_{\mathrm{sc}} \rho_{\mathrm{sc}}\left(c_{\mathrm{sc}}\right.$ and $\rho_{\mathrm{sc}}$ are the silica heat capacity and density) and $\beta_{\mathrm{sc}}$ is the associated heat conductance. Straightforward calculations lead to the following spatial-temporal distribution of the heat delivered by single pulse (cf. (S2)):

$$
\delta \mathrm{T}_{\text {pulse }}(r, t)=\delta \mathrm{T}_{\text {pulse }}(0,0) \pi r\left(\operatorname{erf}\left(\frac{r+R_{\text {eff }}}{2 \sqrt{\kappa t}}\right)-\operatorname{erf}\left(\frac{r-R_{\text {eff }}}{2 \sqrt{\kappa t}}\right)\right)+2 \sqrt{\pi} \sqrt{\kappa t}\left(e^{-\frac{\left(r+R_{\text {eff }}\right)^{2}}{4 \kappa t}}-e^{-\frac{\left(r-R_{\text {eff }}\right)^{2}}{4 \kappa t}}\right),
$$

where $\kappa=\beta_{\text {sc }} / \alpha_{\text {sc }}$. Substituting (S4) into (S3) we obtain the estimate $3<\xi_{\mathrm{II}}<40$ at the hottest point $(r=0)$. Note that this estimate implies ideal thermal contact between the silica spheres and the silicon wafer base, which might not be the case. Hence, the actual value of $\xi_{\text {II }}$ might be even higher than 40.

From comparison of $\xi_{\mathrm{I}}$ and $\xi_{\mathrm{II}}$ we can conclude that the heat extraction along the silver film is likely to dominate the heat leakage into the substrate. Hence, we can estimate the upper bound of heating caused by the train of femtosecond laser pulses as $\Delta \mathrm{T}<\xi_{\mathrm{I}} \delta T_{\text {pulse }} \sim 60 \mathrm{~K}$. 